\title{
Texting bans and fatal accidents on roadways: Do they work? Or do drivers just react to announcements of bans? \\ Rahi Abouk, Scott Adams \\ Online Appendix
}

\section{Table A1}

Effects of texting bans by state

\begin{tabular}{|c|c|c|c|}
\hline Arkansas & $\begin{array}{c}0.0475 \\
(0.0768)\end{array}$ & New Hampshire & $\begin{array}{c}0.4732 \\
(0.1399)^{* * *}\end{array}$ \\
\hline California & $\begin{array}{c}-0.0991 \\
(0.0420) * *\end{array}$ & New Jersey & $\begin{array}{c}-0.1941 \\
(0.0823)^{* *}\end{array}$ \\
\hline Colorado & $\begin{array}{c}0.0137 \\
(0.1047)\end{array}$ & New York & $\begin{array}{c}0.1829 \\
(0.0761)^{* *}\end{array}$ \\
\hline Connecticut & $\begin{array}{l}-0.3754 \\
(0.3552)\end{array}$ & North Carolina & $\begin{array}{l}-0.1799 \\
(0.1136)\end{array}$ \\
\hline Georgia & $\begin{array}{c}0.0119 \\
(0.0679)\end{array}$ & Oregon & $\begin{array}{c}-0.3380 \\
(0.1204)^{* * *}\end{array}$ \\
\hline Illinois & $\begin{array}{c}0.0011 \\
(0.1092)\end{array}$ & Rhode Island & $\begin{array}{c}0.3523 \\
(0.1825)^{*}\end{array}$ \\
\hline Indiana & $\begin{array}{c}0.0571 \\
(0.0721)\end{array}$ & Tennessee & $\begin{array}{c}-0.0150 \\
(0.0810)\end{array}$ \\
\hline Louisiana & $\begin{array}{c}-0.1759 \\
(0.0732)^{* *}\end{array}$ & Utah & $\begin{array}{l}-0.0662 \\
(0.1538)\end{array}$ \\
\hline Maryland & $\begin{array}{l}-0.0391 \\
(0.0836)\end{array}$ & Vermont & $\begin{array}{c}0.2945 \\
(0.2749)\end{array}$ \\
\hline Massachusetts & $\begin{array}{c}0.0342 \\
(0.2252)\end{array}$ & Virginia & $\begin{array}{c}-0.0744 \\
(0.0809)\end{array}$ \\
\hline Michigan & $\begin{array}{c}0.3271 \\
(0.0778)^{* * * *}\end{array}$ & Washington & $\begin{array}{c}0.0828 \\
(0.0666)\end{array}$ \\
\hline Minnesota & $\begin{array}{l}-0.1494 \\
(0.1089)\end{array}$ & Wisconsin & $\begin{array}{c}-0.9781 \\
(0.0743)^{* * *}\end{array}$ \\
\hline Missouri & $\begin{array}{c}0.1042 \\
(0.0822)\end{array}$ & Wyoming & $\begin{array}{c}0.4859 \\
(0.1394)^{* * *}\end{array}$ \\
\hline Nebraska & $\begin{array}{c}0.0119 \\
(0.1045)\end{array}$ & & \\
\hline \multicolumn{4}{|c|}{$\begin{array}{l}\text { Note: Reported are coefficients on an interaction of the texting ban variable with state dummies. The } \\
\text { dependent variable is the natural logarithm of the number of fatal accidents plus one. Newey-West (1987) } \\
\text { standard errors are reported in parentheses correcting for heteroskedasticity and allowing autocorrelation up } \\
\text { to one lag. Each regression includes } 49 \text { states and } 48 \text { month dummy variables, as well as controls listed in } \\
\text { Table } 2 \text { and used in the third column of Table } 3 \text {. } \\
* * * \text { Significant at the } 1 \text { percent level. } \\
* * \text { Significant at the } 5 \text { percent level. } \\
* \text { Significant at the } 10 \text { percent level. }\end{array}$} \\
\hline
\end{tabular}




\section{Table A2}

Effects in selected states

\begin{tabular}{lcc}
\hline Accidents & $\begin{array}{c}\text { Single vehicle, } \\
\text { Single occupant }\end{array}$ & All crashes \\
\hline $\begin{array}{l}\text { California } \\
\text { (vs. Arizona, Nevada, and Oregon) }\end{array}$ & -0.1395 & -0.0148 \\
& $(0.1080)$ & $(0.0500)$ \\
$\begin{array}{l}\text { Louisiana } \\
\text { (vs. Arkansas, Mississippi, and Texas) }\end{array}$ & -0.1128 & -0.0637 \\
Minnesota & $(0.0695)$ & $(0.0423)$ \\
(vs. Iowa and Wisconsin) & -0.0429 & 0.0574 \\
Washington & $(0.2263)$ & $(0.0832)$ \\
(vs. Idaho and Oregon) & 0.0090 & 0.1093 \\
\end{tabular}

Note: Each cell is from a separate regression. Reported are coefficients from a weighted least squares regression, weighted by state population size. The dependent variable is the natural logarithm of the number of fatal accidents plus one. Robust standard errors are in parentheses. Each regression includes state and month dummy variables, as well as controls listed in Table 2 and used in the third column of Table 3 . The control states are those chosen by HLDI (2010) 
Table A3

Additional estimates of the effect of texting bans, with robustness checks

\begin{tabular}{|c|c|c|c|c|}
\hline & & $\begin{array}{l}\text { With state } \\
\text { dummies }\end{array}$ & $\begin{array}{l}\text { With state- } \\
\text { specific trends }\end{array}$ & With both \\
\hline (1) & Preferred Table 3 estimates & $\begin{array}{c}-0.0764 \\
(0.0252) * * *\end{array}$ & $\begin{array}{l}-0.0712 \\
(0.0445)\end{array}$ & $\begin{array}{l}-0.0253 \\
(0.0414)\end{array}$ \\
\hline (2) & $\begin{array}{l}\text { Different weights and population sizes } \\
\text { Unweighted OLS }\end{array}$ & $\begin{array}{l}-0.0099 \\
(0.0493)\end{array}$ & $\begin{array}{r}-0.0147 \\
(.0696)\end{array}$ & $\begin{array}{c}0.0149 \\
(0.0644)\end{array}$ \\
\hline (3) & $\begin{array}{l}\text { Unweighted OLS for states with at least } \\
2 \text { million residents } \\
\text { (drops } 14 \text { small states; total of } 35 \text { ) }\end{array}$ & $\begin{array}{c}-0.0945 \\
(0.0378) * *\end{array}$ & $\begin{array}{l}-0.1212 \\
(0.0624)^{*}\end{array}$ & $\begin{array}{l}-0.0929 \\
(0.0559)\end{array}$ \\
\hline (4) & $\begin{array}{l}\text { Unweighted OLS for states with at least } \\
\text { one accident in every month } \\
\text { (drops } 12 \text { small states; total of } 37 \text { ) }\end{array}$ & $\begin{array}{l}-0.0780 \\
(0.0456)^{*}\end{array}$ & $\begin{array}{l}-0.0935 \\
(0.0756)\end{array}$ & $\begin{array}{l}-0.0625 \\
(0.0677)\end{array}$ \\
\hline (5) & $\begin{array}{l}\text { Poisson for states with at least one } \\
\text { accident in every month }\end{array}$ & $\begin{array}{c}-0.0613 \\
(0.0300)^{* *}\end{array}$ & $\begin{array}{l}-0.0648 \\
(0.0459)\end{array}$ & $\begin{array}{l}-0.0198 \\
(0.0425)\end{array}$ \\
\hline (6) & $\begin{array}{l}\text { Negative binomial for states with at least one } \\
\text { accident in every month }\end{array}$ & $\begin{array}{c}-0.0606 \\
(0.0311)^{*}\end{array}$ & $\begin{array}{l}-0.0714 \\
(0.0499)\end{array}$ & $\begin{array}{l}-0.0226 \\
(0.0434)\end{array}$ \\
\hline (7) & $\frac{\text { Removing questionable states from }}{\text { Control group }}$ & $\begin{array}{c}-0.0838 \\
(0.0261)^{* * *}\end{array}$ & $\begin{array}{c}-0.0803 \\
(0.0459)^{*}\end{array}$ & $\begin{array}{l}-0.0340 \\
(0.0434)\end{array}$ \\
\hline (8) & $\begin{array}{l}\text { New Mexico because of Albuquerque, } \\
\text { Las Cruces, and Santa Fe }\end{array}$ & $\begin{array}{c}-0.0766 \\
(0.0253)^{* * *}\end{array}$ & $\begin{array}{l}-0.0713 \\
(0.0445)\end{array}$ & $\begin{array}{l}-0.0257 \\
(0.0414)\end{array}$ \\
\hline (9) & $\begin{array}{l}\text { Alternative estimations } \\
\text { Balanced set of states with laws in effect for } \\
\text { at least } 6 \text { months }\end{array}$ & $\begin{array}{c}-0.0611 \\
(0.0256)^{* *}\end{array}$ & $\begin{array}{l}-0.0478 \\
(0.0435)\end{array}$ & $\begin{array}{c}0.0041 \\
(0.0404)\end{array}$ \\
\hline
\end{tabular}

Note: See Table 4 notes. 


\section{Table A4}

Full Lead and lag specifications

\begin{tabular}{|c|c|c|c|c|}
\hline & \multicolumn{2}{|c|}{ Without state-specific trends } & \multicolumn{2}{|c|}{ With state-specific trends } \\
\hline & Weak bans & Strong bans & Weak bans & Strong bans \\
\hline Lead 5 & $\begin{array}{c}0.0097 \\
(0.0373)\end{array}$ & $\begin{array}{l}-0.0001 \\
(0.0591)\end{array}$ & $\begin{array}{c}0.0825 \\
(0.0374)^{* *}\end{array}$ & $\begin{array}{c}0.0259 \\
(0.0598)\end{array}$ \\
\hline Lead 4 & $\begin{array}{c}-0.1834 \\
(0.0967)^{*}\end{array}$ & $\begin{array}{l}-0.0282 \\
(0.0699\end{array}$ & $\begin{array}{l}-0.1028 \\
(0.0861)\end{array}$ & $\begin{array}{c}0.0011 \\
(0.0653)\end{array}$ \\
\hline Lead 3 & $\begin{array}{c}0.0982 \\
(0.0722)\end{array}$ & $\begin{array}{l}-0.0031 \\
(0.0576)\end{array}$ & $\begin{array}{c}0.1833 \\
(0.0897)^{* *}\end{array}$ & $\begin{array}{c}0.0295 \\
(0.0571)\end{array}$ \\
\hline Lead 2 & $\begin{array}{c}0.0396 \\
(0.1191)\end{array}$ & $\begin{array}{c}0.0210 \\
(0.0593)\end{array}$ & $\begin{array}{c}0.1304 \\
(0.1434)\end{array}$ & $\begin{array}{c}0.0566 \\
(0.0601)\end{array}$ \\
\hline Lead 1 & $\begin{array}{c}0.1375 \\
(0.0626)^{* *}\end{array}$ & $\begin{array}{c}0.0908 \\
(0.0636)\end{array}$ & $\begin{array}{c}0.2404 \\
(0.0477)^{* * *}\end{array}$ & $\begin{array}{c}0.1291 \\
(0.0699)^{*}\end{array}$ \\
\hline P-value leads & 0.04 & 0.83 & $<0.01$ & 0.54 \\
\hline Lag 1 & $\begin{array}{c}-0.1385 \\
(0.0787)^{*}\end{array}$ & $\begin{array}{l}-0.1733 \\
(0.1092)\end{array}$ & $\begin{array}{c}-0.1362 \\
(0.0825)^{*}\end{array}$ & $\begin{array}{l}-0.1754 \\
(0.1074)\end{array}$ \\
\hline Lag 2 & $\begin{array}{c}0.0357 \\
(0.1523)\end{array}$ & $\begin{array}{l}-0.1382 \\
(0.0939)\end{array}$ & $\begin{array}{c}0.0404 \\
(0.1595)\end{array}$ & $\begin{array}{l}-0.1367 \\
(0.0905)\end{array}$ \\
\hline Lag3 & $\begin{array}{l}-0.0789 \\
(0.0955)\end{array}$ & $\begin{array}{l}-0.0729 \\
(0.1318)\end{array}$ & $\begin{array}{l}-0.0717 \\
(0.1001)\end{array}$ & $\begin{array}{l}-0.0671 \\
(0.1296)\end{array}$ \\
\hline Lag4 & $\begin{array}{l}-0.0052 \\
(0.0933)\end{array}$ & $\begin{array}{c}0.0014 \\
(0.1118)\end{array}$ & $\begin{array}{c}0.0062 \\
(0.0918)\end{array}$ & $\begin{array}{c}0.0133 \\
(0.1103)\end{array}$ \\
\hline Lag 5 & $\begin{array}{c}0.1128 \\
(0.0676)^{*}\end{array}$ & $\begin{array}{l}-0.0619 \\
(0.0935)\end{array}$ & $\begin{array}{c}0.1654 \\
(0.0696)^{* *}\end{array}$ & $\begin{array}{c}0.0040 \\
(0.0889)\end{array}$ \\
\hline Log of population & \multicolumn{2}{|c|}{$\begin{array}{c}1.4408 \\
(1.1378)\end{array}$} & \multicolumn{2}{|c|}{$\begin{array}{c}2.5868 \\
(1.4878)^{* *}\end{array}$} \\
\hline $\begin{array}{l}\text { Log of unemployment } \\
\text { rate }\end{array}$ & \multicolumn{2}{|c|}{$\begin{array}{l}-0.0753 \\
(0.1161)\end{array}$} & \multicolumn{2}{|c|}{$\begin{array}{c}0.3490 \\
(0.1732)\end{array}$} \\
\hline Percent male & \multicolumn{2}{|c|}{$\begin{array}{l}-0.0145 \\
(0.0357)\end{array}$} & \multicolumn{2}{|c|}{$\begin{array}{l}-0.06077 \\
(0.0491)\end{array}$} \\
\hline Log of gas tax & \multicolumn{2}{|c|}{$\begin{array}{l}-0.0486 \\
(0.0825)\end{array}$} & \multicolumn{2}{|c|}{$\begin{array}{c}0.0430 \\
(0.0758)\end{array}$} \\
\hline Other accidents & \multicolumn{2}{|c|}{$\begin{array}{c}0.1742 \\
(0.0488)^{* * *}\end{array}$} & \multicolumn{2}{|c|}{$\begin{array}{c}0.1586 \\
(0.0497) * * *\end{array}$} \\
\hline
\end{tabular}

Note: Each column is from a separate regression (equation (3)). Each specification includes both 49 state and 48 month fixed effects, as well as controls listed in Table 2 and used in column (2) of Table 3 . The lead and lag coefficients are plotted in Figure 1.

*** Significant at the 1 percent level.

** Significant at the 5 percent level.

* Significant at the 10 percent level. 
Table A5

Tests of significance of leads and lags in alternative models

\begin{tabular}{|c|c|c|c|c|c|c|c|c|c|c|c|c|}
\hline & \multicolumn{6}{|c|}{$\begin{array}{l}\text { Universally applied and primarily } \\
\text { enforced ("'strong") }\end{array}$} & \multicolumn{6}{|c|}{$\begin{array}{l}\text { Limited coverage or secondary } \\
\text { enforcement ("weak") }\end{array}$} \\
\hline & $\begin{array}{l}\text { p-vl. } \\
\text { leads }\end{array}$ & lag 1 & $\operatorname{lag} 2$ & $\operatorname{lag} 3$ & $\operatorname{lag} 4$ & $\operatorname{lag} 5$ & $\begin{array}{l}\text { p-vl. } \\
\text { leads }\end{array}$ & $\operatorname{lag} 1$ & $\operatorname{lag} 2$ & $\operatorname{lag} 3$ & $\operatorname{lag} 4$ & $\operatorname{lag} 5$ \\
\hline \multicolumn{13}{|l|}{ Alternative dependent } \\
\hline $\begin{array}{l}\text { Multiple vehicles or } \\
\text { multiple occupants }\end{array}$ & $<.01$ & $\begin{array}{l}.022 \\
(.041)\end{array}$ & $\begin{array}{l}-.076 \\
(.049)\end{array}$ & $\begin{array}{l}-.009 \\
(.052)\end{array}$ & $\begin{array}{l}.005 \\
(.063)\end{array}$ & $\begin{array}{l}.003 \\
(.044)\end{array}$ & $<.01$ & $\begin{array}{l}-.248 \\
(.077)\end{array}$ & $\begin{array}{l}-.043 \\
(.136)\end{array}$ & $\begin{array}{l}-.137 \\
(.096)\end{array}$ & $\begin{array}{l}-.206 \\
(.102)\end{array}$ & $\begin{array}{l}-.185 \\
(.067)\end{array}$ \\
\hline $\begin{array}{l}\text { Accidents per million } \\
\text { vehicle miles travelled }\end{array}$ & .49 & $\begin{array}{l}-.193 \\
(.108)\end{array}$ & $\begin{array}{l}-.155 \\
(.101)\end{array}$ & $\begin{array}{l}-.118 \\
(.150)\end{array}$ & $\begin{array}{l}.006 \\
(.115)\end{array}$ & $\begin{array}{l}-.033 \\
(.094)\end{array}$ & $<.01$ & $\begin{array}{l}-.180 \\
(.087)\end{array}$ & $\begin{array}{l}.026 \\
(.146)\end{array}$ & $\begin{array}{l}-.104 \\
(.115)\end{array}$ & $\begin{array}{l}-.044 \\
(.078)\end{array}$ & $\begin{array}{l}.126 \\
(.064)\end{array}$ \\
\hline \multicolumn{13}{|l|}{$\frac{\text { Alternative legislation/ }}{\text { enforcement }}$} \\
\hline $\begin{array}{l}\text { Handheld cell phone ban } \\
\text { also in place }\end{array}$ & .09 & $\begin{array}{l}-.312 \\
(.149)\end{array}$ & $\begin{array}{l}-.257 \\
(.099)\end{array}$ & $\begin{array}{l}-.396 \\
(.166)\end{array}$ & $\begin{array}{l}-.129 \\
(.119)\end{array}$ & $\begin{array}{l}-.114 \\
(.103)\end{array}$ & $<.01$ & $\begin{array}{l}-.002 \\
(.116)\end{array}$ & $\begin{array}{l}.257 \\
(.113)\end{array}$ & $\begin{array}{l}.035 \\
(.133)\end{array}$ & $\begin{array}{l}.295 \\
(.118)\end{array}$ & $\begin{array}{l}.413 \\
(.136)\end{array}$ \\
\hline $\begin{array}{l}\text { Handheld ban not in } \\
\text { place }\end{array}$ & .76 & $\begin{array}{l}-.122 \\
(.120)\end{array}$ & $\begin{array}{l}-.067 \\
(.105)\end{array}$ & $\begin{array}{l}.082 \\
(.107)\end{array}$ & $\begin{array}{l}.086 \\
(.121)\end{array}$ & $\begin{array}{l}.093 \\
(.087)\end{array}$ & $<.01$ & $\begin{array}{l}-.181 \\
(.125)\end{array}$ & $\begin{array}{l}-.036 \\
(.246)\end{array}$ & $\begin{array}{l}-.029 \\
(.117)\end{array}$ & $\begin{array}{l}-.079 \\
(.111)\end{array}$ & $\begin{array}{l}.087 \\
(.053)\end{array}$ \\
\hline \multicolumn{13}{|l|}{ Alternative modeling } \\
\hline Negative Binomial & .35 & $\begin{array}{l}-.090 \\
(.067)\end{array}$ & $\begin{array}{l}-.082 \\
(.044)\end{array}$ & $\begin{array}{l}.023 \\
(.041)\end{array}$ & $\begin{array}{l}.006 \\
(.044)\end{array}$ & $\begin{array}{l}.002 \\
(.038)\end{array}$ & .14 & $\begin{array}{l}-.073 \\
(.040)\end{array}$ & $\begin{array}{l}-.031 \\
(.064)\end{array}$ & $\begin{array}{l}-.006 \\
(.035)\end{array}$ & $\begin{array}{l}-.017 \\
(.039)\end{array}$ & $\begin{array}{l}.040 \\
(.020)\end{array}$ \\
\hline \multicolumn{13}{|l|}{ Additional tests } \\
\hline $\begin{array}{l}\text { Removing the control for } \\
\text { state-specific effects }\end{array}$ & .68 & $\begin{array}{l}-.116 \\
(.060)\end{array}$ & $\begin{array}{l}-.075 \\
(.060)\end{array}$ & $\begin{array}{l}.006 \\
(.081)\end{array}$ & $\begin{array}{l}.085 \\
(.075)\end{array}$ & $\begin{array}{l}.052 \\
(.051)\end{array}$ & $<.01$ & $\begin{array}{l}-.017 \\
(.087)\end{array}$ & $\begin{array}{l}.147 \\
(.144)\end{array}$ & $\begin{array}{l}.035 \\
(.071)\end{array}$ & $\begin{array}{l}.111 \\
(.101)\end{array}$ & $\begin{array}{l}.266 \\
(.068)\end{array}$ \\
\hline $\begin{array}{l}\text { Balanced set of states } \\
\text { with laws in effect for at } \\
\text { least } 6 \text { months }\end{array}$ & .02 & $\begin{array}{c}-.174 \\
(.104)\end{array}$ & $\begin{array}{l}-.164 \\
(.092)\end{array}$ & $\begin{array}{l}-.065 \\
(.136)\end{array}$ & $\begin{array}{c}.009 \\
(.112)\end{array}$ & $\begin{array}{l}-.005 \\
(.089)\end{array}$ & $<.01$ & $\begin{array}{l}-.132 \\
(.087)\end{array}$ & $\begin{array}{c}.063 \\
(.161)\end{array}$ & $\begin{array}{l}-.041 \\
(.098)\end{array}$ & $\begin{array}{c}.023 \\
(.094)\end{array}$ & $\begin{array}{c}.190 \\
(.070)\end{array}$ \\
\hline
\end{tabular}

Note: Each row is from a separate regression estimation of equation (2). Each specification includes state and month fixed effects, as well as controls listed in Table 2 and used in the fifth column of Table 3 . These include state-specific trends. 


\section{Appendix Figure A1}

1a. Universally applied and primarily enforced bans; lags extended to 8 months

Without state-specific trends

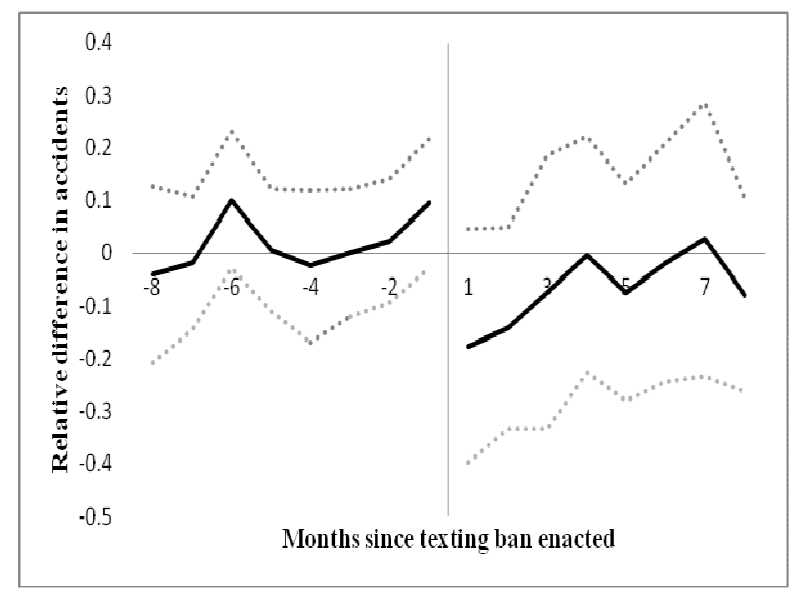

With state-specific trends

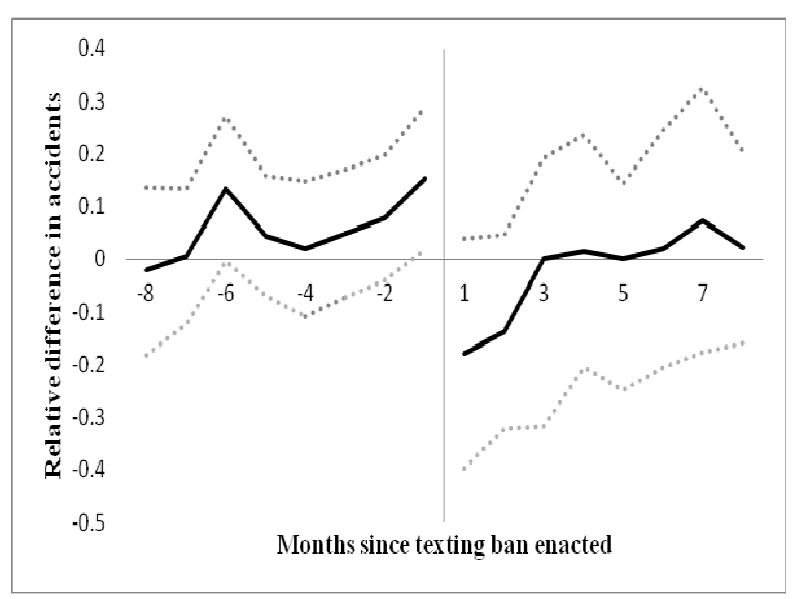

1b. Universally applied and primarily enforced bans; lags extended to 11 months

Without state-specific trends

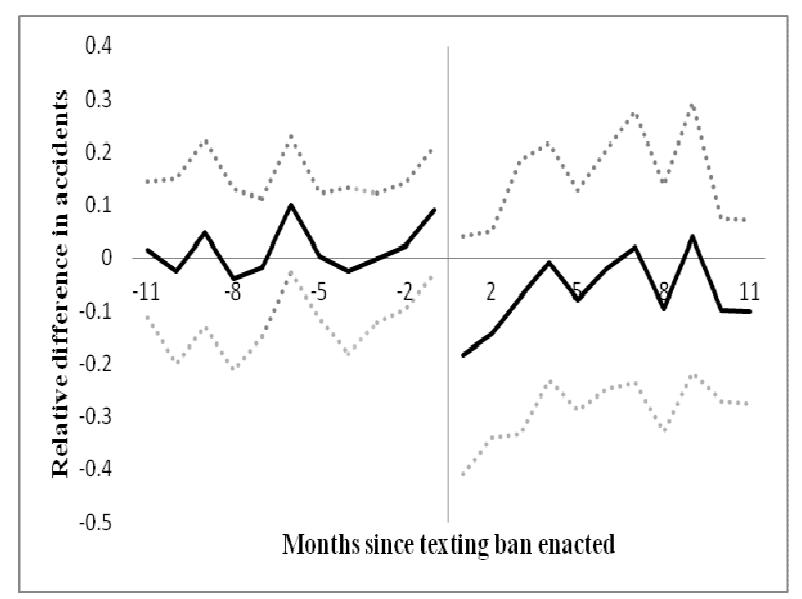

With state-specific trends

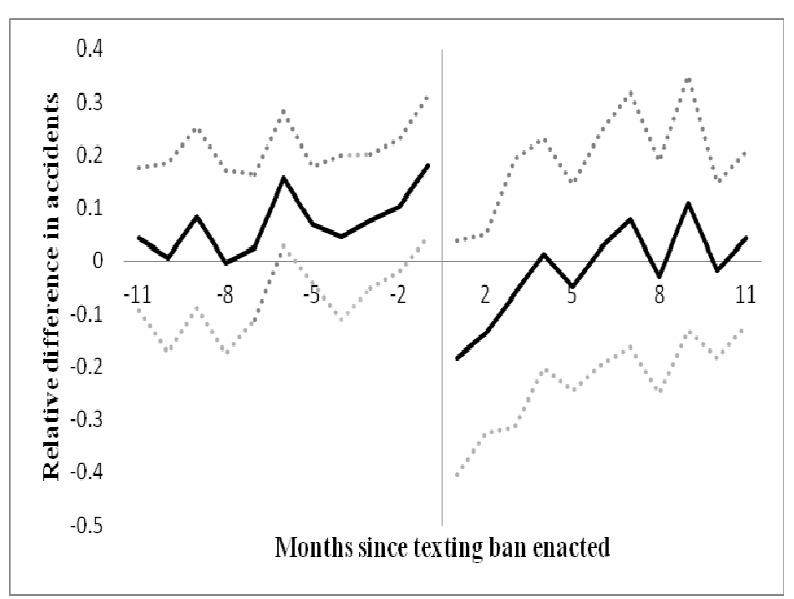

Note: These figures plot the estimated lead and lag coefficients from equation (2) extended to additional leads and lags. Regressions include both 49 state and 48 month fixed effects and the control variables from the third column of Table 3 . 